\title{
Case of Chronic Recycling Toxocararosis Caused by Toxocara cati
}

\author{
Valeri R Velev* \\ Parasitology and Tropical Medicine, Medical University of Sofia, Bulgaria
}

*Corresponding author: Valeri R Velev, Chair of Infectious Diseases, Parasitology and Tropical Medicine, Medical University of Sofia, Bulgaria, Tel: 0889563412; E-mail:velev_md@abv.bg

Received date: June 27, 2017; Accepted date: July 21, 2017; Published date: July 26, 2017

Citation: Valeri RV (2017) Case of Chronic Recycling Toxocararosis Caused by Toxocara cati. Pediatric Infect Dis Vol.2 No.3: 49.

Copyright: (2017 Valeri RV. This is an open-access article distributed under the terms of the Creative Commons Attribution License, which permits unrestricted use, distribution, and reproduction in any medium, provided the original author and source are credited.

\section{Introduction}

Toxocarosis is a parasitic zoonosis caused by dog and cat helminthes, respectively Toxocaracanis and T. cati. Other species of the genus Toxocara are also known, but they have an unproven relationship to human pathology. These helminthes parasite mainly in the intestines, organs and tissues of the dog and cat, but in certain cases they can also be met in a much wider range of hosts-jackals, foxes, wolves, etc. It is important for the epidemiology of the disease that infected animals emitting eggs have no clinical manifestations. Eggs spend different time in the soil under certain ecological conditions before they become invasive for a human or other host [1-4].

Infection in humans is most common by swallowing invasive eggs in poor personal hygiene and, more rarely, by the consumption of larval invaded meat from host animals. According to the discoverer of Beaver's parasitism, the most common are boys between 2 and 7 years of age, with hepatomegaly, eosinophilia, allergic symptoms and a history of contact with dogs. In fact, the most common form of toxocarosis, visceral, occurs with a polymorphic clinical picture, with most visible intoxication with subfebrility and adynamics, pulmonary and gastrointestinal manifestations [5-7]. These features, along with ignorance of parasitosis, lead to serious differential diagnosis difficulties.

According to most literary sources, $T$. canis has a leading role in human infectious pathology, probably due to the widespread spread of ownerless dogs and the fact that female dogs can transfer transplanted larvae into their offspring, unlike cats. In connection with this, it has now been proved that dogs under 1 year have the most serious epidemiological significance. Unlike the countries of Western Europe and most of the countries of Southeastern Europe, there are only partial studies on the seroepidemiology of the disease [3-4]. In some preliminary studies conducted by the Parasitology Division of the National Center for Infectious and Parasitic Diseases (NCIPD), specific antibodies in more than $12 \%$ of those surveyed [3] have been shown in a contingent with clinical manifestations. In comparison, the percentage in the Czech Republic varies between 6-36\%, in Slovakia the healthy population is seropositive in over $13 \%$ of cases, and in countries like Egypt the seropositivity is over $50 \%$ [3-4].
Today there are several working classifications regarding the clinical forms of toxocarosis, but the most used by parasitologists in our country is that of Avudiuhina (2002). She examines the following 5 forms: visceral toxocarosis (affecting organs of digestive, respiratory and excretory systems), ocular toxocarosis, central nervous system toxocarosis, connective tissue toxocarosis and disseminated toxocarosis (usually in immunocompromised). The duration of flow is subdivided into acute and chronic, with chronic phases of exacerbations and remissions. The classic visceral pattern most commonly occurs with pulmonary syndrome-dry cough, asthma-like seizures, and gastrointestinal tract is with nausea, rarely with vomiting, diarrhea, weight in the epigastrium. Usually the patients are afebrile or subfebrile; characteristic are hepatomegaly, eosinophilia and, in some cases, recurrent urticarial rashes. In rare cases, myocarditis, pericarditis, alopecia $[1,3,8]$ have been reported in seropositive individuals.

In terms of diagnostics, the only valid and routinely used methods today are immunological-serological evidence of specific IgM/IgG class antibodies, most often against parasite excretory-secretory antigens. In cases of diagnostic difficulties we include immunoblotting, a technique that verifies serological data. Molecular biological methods are not used in routine diagnosis due to high cost and labor intensity [2-3].

\section{Clinical Case}

This is a 7-year-old child born of a second normal pregnancy who has had an acute illness 4 days before hospitalization with a fever of up to $39^{\circ} \mathrm{C}$, nausea without vomiting and adynamics. An empirical treatment with a penicillin antibiotic has begun following a review by a GP but complaints persist.

In a conversation with the mother became clear that she had been self-directed to the hospital due to the fact that the child's home yard had a cat that was diagnosed by a veterinarian as a parasited with toxocariasis. On this occasion, a child's serological test- $\operatorname{lgG}(+)$, titre 1.6 (positive values $>1.1$ ) was performed a month and a half ago. Etiological treatment of toxocarosis has not been performed due to lack of symptoms.

Upon arrival, the child was in a state of general harm. Contact, adequate. Intoxicated, febrile to $38.2^{\circ} \mathrm{C}$. Skin-pale, clean, without rash. No peripheral lymphodulopathy. Throat- 
moderately hyperemised. Tongue-dry, furred. With pure bilateral vesicular breathing, and cardiac activity with normal frequency. Abdomen-soft, with no palpable soreness, lively intestinal peristalsis. Liver at $1.5-2.0 \mathrm{~cm}$ under a ribbed arc, with elastic consistency and sensitive to palpation; spleen $+1 \mathrm{~cm}$. Succusio renalis-bilateraly negative. Normal neurological status.

There was no evidence of inflammatory changes from the blood picture, but eosinophilia was established by the manual count of white blood cells -St 20\%; Sg 32\%; Mo 0.7\%; Eo $41 \%$; No evidence of hepatic or renal involvement was seen from the biochemical parameters.

Lung X-ray showed a normal illumination of the two lung fields, and abdominal echography-a mild hepatomegaly. From the serological test for toxocarosis we made an increased titer of specific antibodies-lgG(+), titer 1.8 .

We began symptomatic treatment with venous infusions and antipyretics and etiological treatment with rising doses of Albendazole.

\section{Discussion}

Toxocarosis, though overlooked and poorly known, is not so rare in developed countries as well. Considering that the host is the most common dog and considering the frequency of echinococcosis, another parasitosis associated with it, we should expect a serious incidence of this helminthosonosis. In most literary sources, $T$. cati is considered to be a relatively rare cause of the disease, and attention to prevention and clinical thinking is mainly directed towards dog toxocarosis $[3,6,7]$. In our case, we describe $T$. cati's toxocarosis, albeit with an indirectly proven etiological connection.

Based on the anamnesis-adynamia and hypotension, persistent fever, and specific epidemiological history; status and serological tests-we have accepted that it is a severe form of toxocarosis, probably in the course of chronic recurrent, untreated etiological parasitosis.
In a large number of cases, toxocarosis can occur both subclinically, non-invariably. This is especially common in children. Clinical signs are nonspecific, "deleted"- adynamics, indigestion or capricious appetite, subfebrility, nausea, recurrent diarrhea, rapid transient rash, asthma-like attacks [3,5]. Any case of eosinophilia, unclear febrile state or unspecified rash syndrome in combination with hepatomegaly and a suitable epidemiological history should also be considered in the direction of toxocarosis. In conclusion, some authors believe that the deworming is important and should be indicated at least twice a year, having drugs albendazole or mebendazole [9].

\section{References}

1. Avduhina I (2002) Toxocarosis. Clinical Parasitology 1: 499-514.

2. Rainova I (2001) Algorithm in the diagnosis of toxocarosis. Congress of Infectious and Parasitic diseases and Epidemiology, St.Zagora, 1013V Collection of abstracts 34-35.

3. Rainova I (2008) Toxocarosis, 2008, 1st edition, Sofia, "Pensoft".

4. Rainova I, Kurdova R (2007) Contemporary state and clinicaldiagnostic problems of toxocarosis in people in Bulgaria. Infectology 1: 137-138.

5. Yaneva P, Hadjiiski P, Shentova R (2015) Toxocarosis - a diagnostic case and a challenge for the doctor. Clinical case and literary review. Pediatrics 3: 54-56.

6. Beaver C (1954) Parasitic diseases of animals and their relation to public health. Small Animal Practice 49: 199-205.

7. Eberhard M, Alfano E (1998) Adult Toxocara cati infection in U.S children: report of four cases. Am J Trop Med Hyg 59: 404-406.

8. Oteifa M, Moustafa M, Elgozamy B (1998)Toxocariasis as a possible cause of allergic diseases in children. J Egypt Soc Parasitol 28: $365-372$

9. Woodhall D, Fiore A (2014) Toxocariasis: A Review for Pediatricians. J Pediatric Infect Dis Soc 3: 154-9. 